\title{
Channel Modeling and Analysis of Reconfigurable Intelligent Surfaces Assisted Vehicular Networks
}

\author{
Long Kong ${ }^{\dagger}$, Jiguang $\mathrm{He}^{b}$, Yun $\mathrm{Ai}^{\ddagger}$, Symeon Chatzinotas ${ }^{\dagger}$, and Björn Ottersten ${ }^{\dagger}$ \\ ${ }^{\dagger}$ The Interdisciplinary Centre for Security Reliability and Trust (SnT), University of Luxembourg, Luxembourg \\ ${ }^{b}$ Center for Wireless Communications (CWC), University of Oulu, Oulu, Finland \\ ${ }^{\ddagger}$ Faculty of Engineering, Norwegian University of Science and Technology, 2815 Gjøvik, Norway \\ Emails: long.kong@uni.lu, jiguang.he@oulu.fi,yun.ai@ntnu.no, symeon.chatzinotas@uni.lu, bjorn.ottersten@uni.lu
}

\begin{abstract}
The new concept named reconfigurable intelligent surfaces (RIS) is becoming an appealing enabler due to its uniqueness with having low hardware complexity and low power consumption advantages simultaneously. In this paper, an RISaided vehicular Adhoc network (VANET) is considered, where the beacon vehicle is enabled with a passive RIS, the communication links between the beacon vehicle and client vehicle caused due to the multipath fading effects, are modeled with Fox's $H$-function distribution. This paper first models the inter-vehicle links for the given system setup and then investigates the outage probability and effective rate as performance metrics. More specifically, the unsupervised expectation-maximization (EM) algorithm is consequently used to characterize the distribution of the received signal-to-noise ratio (SNR) at the client vehicle, which is modeled as the mixture of Gaussian (MoG) distribution. The accuracy of our approach is further validated with the Kolmogorov-Smirnov (KS) goodness of fit test. The MoG-based approach successfully tackles the RIS-enabled inter-vehicle communication with an easy, accurate, and tractable solution compared to the widely used central limit theorem (CLT) method. It leads to the closedform outage probability and effective rate expressions.

Index Terms-Reconfigurable intelligent surfaces (RIS), mixture of Gaussian (MoG) distribution, unsupervised expectationmaximization (EM) machine learning, effective rate, Fox's $H$ function, inter-vehicle communications.
\end{abstract}

\section{INTRODUCTION}

Recently, reconfigurable intelligent surfaces (RIS)-based wireless transmission is rapidly considered and explored in various wireless communications scenarios, e.g., deviceto-device communications [1], millimeter-wave (mmWave) multiple-input multiple-output (MIMO) system [2], [3], covert communication [4], physical layer security [5], [6], index modulation [7], smart cities [8], etc. What makes the RIS technology popular lies in its unique capability in controlling the propagation environment so as to increase the quality of service (QoS) [5], [7], [9]-[12].

An RIS is essentially an electronically operated metasurface controlled by programmable software, which is physically equivalent to digitally controllable scatterers and softwaredefined surface [4], [13]. A large number of small, low-cost, and passive artificial 'meta-atoms' integrated in an RIS can smartly change the reflection direction towards any desired users by tuning a series of phase shifters [7]. Compared to relaying technique, the RIS technology does not require any power amplifier to achieve coverage extension. Besides, the RIS structures can be easily deployed into factories, buildings, rooms, vehicles, etc. Centered on the RIS-enabled communication scenarios, numerous works have investigated the benefits brought by the RIS technique in a diverse set of wireless communication scenarios [7], [9], [14]-[18].

On selected RIS-enabled scenarios, Makarfi et al. in [5] studied the physical layer security of RIS-aided vehicle networks, where the inter-vehicle links are modeled with Rayleigh fading. It is noteworthy to mention that Jeong et al. in [19] proposed to utilize Fox's $H$-function distribution to model the pathloss, fading, and shadowing of inter-vehicle links. More importantly, this work successfully validated the usage of Fox's $H$-function to fit the experimental measurement at $5 \mathrm{GHz}$ and $5.2 \mathrm{GHz}$. The Fox's $H$-function distribution ${ }^{1}$ has been widely regarded as a flexible and general model, which can broadly subsume many well-known fading models, e.g., Rayleigh, Nakagami- $m$, Gamma, $\alpha-\mu$, Fisher-Snedecor $\mathcal{F}$, etc [21]-[24]. To quantify the statistical QoS of RIS-assisted wireless communication systems, the authors in [25] studied the effective capacity over Rayleigh fading channels. To the authors' best knowledge, the RIS-aided inter-vehicle communication has not been investigated yet.

To this end, the core focus of this paper is to study the RIS-enabled inter-vehicle communications using Fox's $H$ function distribution to model the channel characteristics. To evaluate the investigated system configuration performance, performance metrics such as outage probability and effective rate are chosen (see [26]). The contribution of this paper are summarized as follows:

1) Different from the existing works, where the propagation environment of inter-vehicle communication is modeled by Rayleigh/Nakagami-m/Rician distribution, we apply a more general model, i.e., the Fox's $H$-function distribution, to model the multipath effect in the RIS-enabled inter-vehicle communications.

2) Considering the RIS-assisted inter-vehicle communications, the characteristics of the received signal-to-noise ratio (SNR) at the client vehicle is approximated with

${ }^{1}$ In addition, the Fox's $H$-function distribution is also studied in many other realistic scenarios, e.g., molecular communication [20], inter-vehicle communications [19], wireless communications [21], etc. 


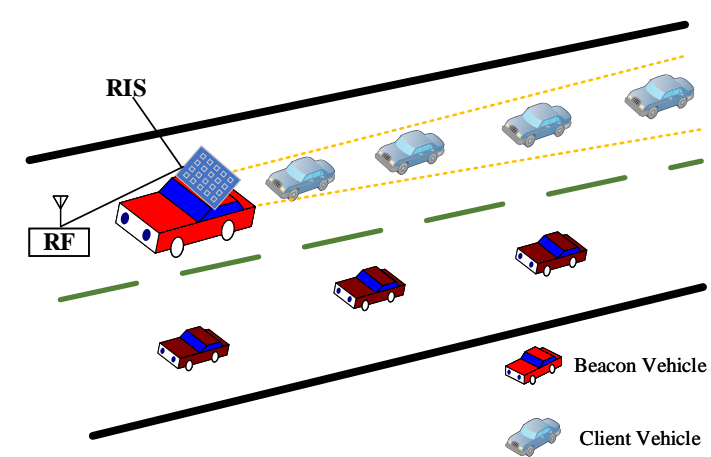

Fig. 1: An RIS-assisted inter-vehicle communications system.

the unsupervised expectation-maximization (EM)-based mixture of Gaussian $(\mathrm{MoG})^{2}$ distribution rather than using the well-reported central limit theorem (CLT) method [5], [7], an easy and highly accurate solution is obtained. The MoG-based solution's accuracy is further confirmed with the Kolmogorov-Smirnov (KS) goodness of fit test.

3) Outage probability and effective rate, being our performance evaluation metrics, are approximated with closedform expressions, and their tightness is validated with Monte-Carlo simulations.

4) When Fox's $H$-function distribution is reduced to Rayleigh distribution, the closed-form expressions of the outage probability with the CLT- and MoG- based approaches are further compared. Numerical results demonstrate that the MoG-based solution always provides a good solution for a small or large number of the reflectors on an RIS.

The rest of this paper is structured as follows. In Section II, we introduce the system model and formulate the effective rate as one of the performance metrics. Section III, we mainly characterize the outage probability and effective rate analysis. Section IV presents the numerical results and useful insights. Finally, concluding remarks are summarized in Section V.

\section{SYSTEM MODEL AND PROBLEM FORMULATION}

\section{A. System Model}

Consider a vehicular network, as shown in Fig. 1, where safety messages are sent from the beacon (head) vehicle operating in a sectorized transmission mode to the client vehicles. The beacon vehicle works on the RIS-AP ${ }^{3}$ scheme, namely, the head vehicle transmits the safety messages with the help of an RIS [7]. The RIS is composed of $N$ passive and lowcost reflecting elements. The safety messages generated by a nearby radio-frequency $(\mathrm{RF})$ source in a deliberate manner are reflected in the direction of a target client vehicle.

\footnotetext{
${ }^{2}$ The 'MoG' is also known as Gaussian mixture model (GMM) in machine learning area [27].

${ }^{3}$ The scenario of the RIS-aided transmitter being an access point (AP) has been widely reported in the open literature, see references [5], [7], [12].
}

For the given system model, the received signal at the client vehicle whilst ignoring the fading between the RF source and the RIS is given as

$$
y=\sqrt{E_{s}} \sum_{n=1}^{N} g_{n} \exp \left(j \psi_{n}\right) s+w,
$$

where $j=\sqrt{-1}, E_{s}$ is the transmit power, $g_{n}=h_{n} e^{-j \phi_{n}}$ is the wireless fading channel between the beacon vehicle to the client vehicle, $h_{n}$ is the amplitude of the fading channel. $\phi_{n}$ is the phase of the fading channel gain. $\psi_{n}$ is the adjustable phase induced by the $n$-th reflecting meta-surface of the RIS. $s$ is the transmitted signal with unit energy, $w$ is the additive white Gaussian noise (AWGN) with zero mean and $N_{0}$ variance.

According to [19, Sec. III], the Fox's $H$-function distribution is adopted to characterize $h_{n}$ with the probability density function (PDF) $f_{h}(x)$, which is expressed as

$$
\begin{aligned}
& f_{h}(x)=\mathcal{K} H_{p, q}^{m, n}\left[\mathcal{C} x \mid \begin{array}{c}
\left(a_{i}, A_{i}\right)_{i=1: p} \\
\left(b_{l}, B_{l}\right)_{l=1: q}
\end{array}\right] \\
& =\frac{\mathcal{K}}{2 \pi j} \int_{\mathcal{L}} \frac{\prod_{l=1}^{m} \Gamma\left(b_{l}+B_{l} s\right) \prod_{i=1}^{n} \Gamma\left(1-a_{i}-A_{i} s\right)(\mathcal{C} x)^{-s}}{\prod_{l=m+1}^{q} \Gamma\left(1-b_{l}-B_{l} s\right) \prod_{i=n+1}^{p} \Gamma\left(a_{i}+A_{i} s\right)} d s,
\end{aligned}
$$

where $\mathcal{C}>0$ and $\mathcal{K}$ are constants such that $\int_{0}^{\infty} f(\gamma) d \gamma=1$. $A_{i}>0$ for all $i=1, \cdots, p$, and $B_{l}>0$ for all $l=1, \cdots, q$. $0 \leq m \leq q, 0 \leq n \leq p, \mathcal{L}$ is a suitable contour separating the poles of the gamma functions $\Gamma\left(b_{l}+B_{l} s\right)$ from the poles of the gamma functions $\Gamma\left(1-a_{i}-A_{i} s\right)$. For the simplicity of notations, the Fox's $H$-function distribution is denoted as $H_{p, q}^{m, n}(\mathbb{P})$, where $\mathbb{P}=(\mathcal{K}, \mathcal{C}, \mathfrak{a}, \mathfrak{b}, \mathscr{A}, \mathscr{B})$, $\mathfrak{a}=$ $\left(a_{1}, \cdots, a_{p}\right), \mathscr{A}=\left(A_{1}, \cdots, A_{p}\right), \mathfrak{b}=\left(b_{1}, \cdots, b_{q}\right)$, and $\mathscr{B}=\left(B_{1}, \cdots, B_{q}\right)$

From (1), the received instantaneous SNR at the client vehicle is

$$
\gamma=\frac{E_{s}}{N_{0}}\left|\sum_{n=1}^{N} h_{n} e^{j\left(\phi_{n}-\psi_{n}\right)}\right|^{2} .
$$

Considering the best case at the client vehicle, i.e., to maximize the instantaneous SNR $\gamma$, the channel phases from the intelligent surface are accordingly eliminated; in other words, $\phi_{n}=\psi_{i}$ [7], [9], [15]. The received instantaneous SNR can be written as

$$
\gamma=\rho\left|\sum_{n=1}^{N} h_{n}\right|^{2}
$$

where $\rho=\frac{E_{s}}{N_{0}}$. For the simplicity of notation, let $Y=$ $\sum_{n=1}^{N} h_{n}$. The PDF of $Y$ is actually the PDF of the sum of $N$ independent Fox's $H$ distributed random variable $h_{n}$. As indicated in [22], the PDF of the sum of $N$ independent Fox's $H$ variate is expressed in terms of the multivariate Fox's $H$-function. In order to obtain a simple and useful PDF of $\gamma$, the MoG distribution is adopted as a highly tight and flexible alternative to approximate the exact PDF of $Y$. The details of the MoG distribution will be introduced in the next Section. 


\section{B. Problem Formulation}

As talked in the Introduction, the RIS technology is a desirable candidate to boost the QoS. One metric to demonstrate the statistical QoS is the effective rate, and it is defined as the maximum constant arrival rate that a time-varying service process can support with statistical latency guarantees [28], [29]. It acts as a joint mathematical framework, connecting the physical layer and link layer, and is used to explore the performance of various wireless networks under certain delay constraints [29]. The concept of effective rate has been widely applied to investigate the trade-off among latency, reliability, energy efficiency, and security. The normalized effective rate over the instantaneous received SNR $\gamma, \gamma \geq 0$ with the average SNR $\rho$ is defined as [28]

$$
\mathcal{R}=-\frac{1}{A} \log _{2}[\underbrace{\mathbb{E}\left((1+\gamma)^{-A}\right)}_{\mathcal{U}}] \text { bits/s/Hz, }
$$

where $A=\frac{\theta T B}{\ln 2} . \mathbb{E}[x]$ denotes the expectation operator over a random variable $x . \theta$ is the delay quality of service (QoS) exponent, $T$ is the block-length, and $B$ denotes the bandwidth of the system. The QoS exponent $\theta$ is defined from the delay outage probability, which is mathematically formulated as

$$
\theta=-\lim _{Q_{\max } \rightarrow \infty} \frac{\ln \left(\operatorname{Pr}\left(Q \geq Q_{\max }\right)\right)}{Q_{\max }},
$$

where $\operatorname{Pr}(a \geq b)$ stands for the probability of $a$ being larger than or equal to $b$. Herein, $Q$ denotes the queue length at the transmitter buffer side, and $Q_{\max }$ is the predetermined threshold of the queue length. Practically speaking, $\theta \rightarrow 0$ implies the delay-tolerant communication, while $\theta \rightarrow \infty$ implies the delay-limited communication [30], e.g., voice calls.

\section{EFFECTIVE RATE CHARACTERIZATION}

\section{A. Preliminary}

To provide a closed-form effective rate expression, the PDF of $\gamma$ is essential for the derivations. In this section, we have applied the MoG distribution to approximate the PDF of $\gamma$. As stated in [31], the MoG distribution can be used to model any arbitrarily shaped non-Gaussian density, e.g., Nakagami/Lognormal, Weibull, $\kappa-\mu, \eta-\mu, \alpha-\mu$, cascaded $\alpha-\mu$ distributions, etc. The intrinsic parameters used to represent the MoG distribution are implemented via the completely unsupervised expectation-maximization (EM) learning algorithm. The MoG distribution is proved highly advantageous in [32] to provide an easy and straightforward solution when analyzing the physical layer security performance over various fading channels. Against this background, we herein offer the MoG distribution with its PDF and cumulative distribution function $(\mathrm{CDF})$.

1) $P D F \& C D F$ : The PDF and $\mathrm{CDF}$ of the MoG distribution are respectively given as

$$
f_{Y}(y)=\sum_{l=1}^{C} \frac{w_{l}}{\sqrt{2 \pi} \eta_{l} \sqrt{\gamma}} \exp \left(-\frac{\left(y-\mu_{l}\right)^{2}}{2 \eta_{l}^{2}}\right),
$$

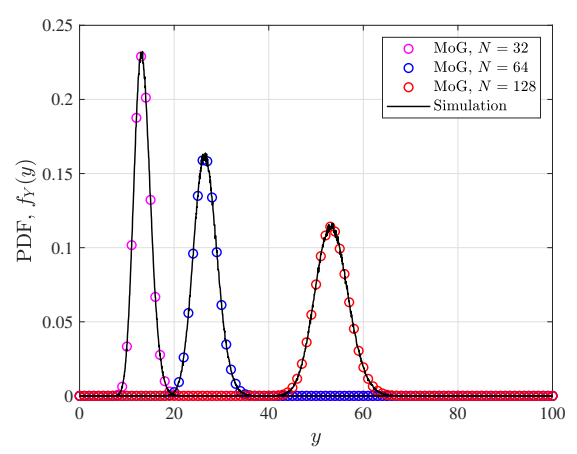

Fig. 2: MoG parameters for $Y$ over Fox's $H$ function fading channels, i.e., $H_{0,3}^{3,0}(\mathbb{P})$, and $\mathbb{P}=(0.225,5.774,[\quad],[\quad],[1.5,0.4,4.5],[0.5,0.5,0.5])$ for selected number of $N$, and $C=5,2,6$.

TABLE I: MOG PARAMETERS FOR $Y$ OVER FOX'S $H$-FUnCtion FAding CHANNELS, $H_{0,3}^{3,0}(\mathbb{P})$, AND $\mathbb{P}=$ $(0.225,5.774,[],[],[1.5,0.4,4.5],[0.5,0.5,0.5])$ FOR $N=$ 128.

\begin{tabular}{|c|c|c|c|}
\hline \hline$l$ & $w_{l}$ & $\mu_{l}$ & $\eta_{l}$ \\
\hline 1 & 0.161146048781517 & 49.9901554965529 & 2.589121500066937 \\
\hline 2 & 0.320911738152015 & 52.9081769211079 & 2.889399121690905 \\
\hline 3 & 0.331216390592880 & 53.6466250754554 & 2.896575145921490 \\
\hline 4 & 0.186725822473589 & 56.5412629864913 & 3.236716833388210 \\
\hline \hline
\end{tabular}

$$
F_{Y}(y)=\sum_{l=1}^{C} w_{l} \Phi\left(\frac{y-\mu_{l}}{\eta_{l}}\right)
$$

where $C$ represents the number of Gaussian components. $w_{l}>$ $0, \mu_{l}$, and $\eta_{l}$ are the $l$ th weight, mean, and variance with the constraint of $\sum_{l}^{C} w_{l}=1 . \Phi(\cdot)$ is the CDF of the standard normal distribution.

As shown in Fig. 2, we have plotted the approximated PDF of $Y=\sum_{n=1}^{N} h_{n}$, where $h_{n}$ follows Fox's $H$-function fading. The MoG parameters for $N=15$ are listed in Table. I. One can observe that the MoG approximation is tight and accurate.

Using the probability theory, the PDF and CDF of $\gamma=\rho Y^{2}$ are thereafter given as follows:

$$
\begin{gathered}
f_{\gamma}(y)=\sum_{l=1}^{C} \frac{w_{l}}{\sqrt{8 \pi \rho} \eta_{l} \sqrt{y}} \exp \left(-\frac{\left(\sqrt{y / \rho}-\mu_{l}\right)^{2}}{2 \eta_{l}^{2}}\right), \\
F_{\gamma}(y)=\sum_{l=1}^{C} w_{l} \Phi\left(\frac{\sqrt{y / \rho}-\mu_{l}}{\eta_{l}}\right) .
\end{gathered}
$$

2) Approximation Accuracy: To evaluate the accuracy of the MoG distribution, the KS goodness of fit test is accordingly conducted [33]. The KS test statistic $D$ is to maximize the difference between the simulated and approximated CDFs, i.e., $D \triangleq \max \left|F_{Y}(y)-F_{\hat{Y}}(y)\right|$, where $F_{Y}(y)$ is the empirical $\mathrm{CDF}$ of $\mathrm{RV} Y$, and $F_{\hat{Y}}(y)$ is the approximated CDF given in (6b). Let the hypothesis $\mathbf{H}_{0}$ denotes that $Y$ follows the 


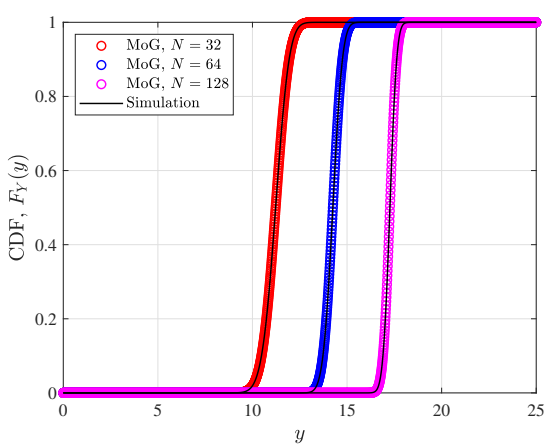

Fig. 3: Analytical and simulated CDF of $\gamma$.

MoG distribution. Such an hypothesis is accepted only when $D$ is smaller than the critical value $D_{\max }$, i.e., $D<D_{\max }$, which is given by $D_{\max } \approx \sqrt{-(1 / 2 v) \ln (\tau / 2)}$, where $\tau$ is the significance level, and $v$ is the number of random samples of $Y$.

Obviously, Fig. 3 presents that the MoG based CDF of $Y$ approximated the Monte-Carlo simulation results with good accuracy. The KS test statistic $D$ obtained from Fig. 3 when $N=32,64,128$ are respectively given as $3.58 \times 10^{-4}, 1.2 \times$ $10^{-3}$, and $7.33 \times 10^{-4}$. Herein, in our KS goodness of fit test, let $\alpha=5 \%$, and $v=10^{6}$. Thus, we have $D_{\max }=0.0014=$ $1.4 \times 10^{-3}$. The hypothesis $\mathbf{H}_{0}$ is surely accepted for the cases $N=32,64,128$ due to $D<D_{\max }$.

\section{B. Effective Rate Characterization}

The effective rate of the RIS-assisted inter-vehicle communications over Fox's $H$-function fading channels is given by

$$
\begin{aligned}
\mathcal{R} \approx & -\frac{1}{A} \log _{2}\left[\sum _ { l = 1 } ^ { C } w _ { l } \left(\frac{1}{6\left(1+\rho\left(\mu_{l}+\sqrt{3} \eta_{l}\right)^{2}\right)^{A}}\right.\right. \\
& \left.\left.+\frac{2}{3\left(1+\rho \mu_{l}^{2}\right)^{A}}+\frac{1}{6\left(1+\rho\left(\mu_{l}-\sqrt{3} \eta_{l}\right)^{2}\right)^{A}}\right)\right] .
\end{aligned}
$$

Proof. Motivated from the MoG based PDF of $\gamma$, i.e., (6a), one can observe that $\gamma$ is modeled in terms of the $C$ normally distributed random variable, i.e., $\gamma=\sum_{l=1}^{C} \gamma_{l}$, and $\gamma_{l} \backsim \mathcal{N}\left(\mu_{l}, \eta_{l}\right)$. Performing a revisit to $\mathcal{U}$ given in (5), and making change of variables $x=\sqrt{\frac{\gamma}{\rho}}$, then substituting (6a) into $\mathcal{U}$, we have

$$
\mathcal{U}=\sum_{l=1}^{C} \frac{w_{l}}{\sqrt{2 \pi} \eta_{l}} \int_{0}^{\infty} \frac{1}{\left(1+\rho x^{2}\right)^{A}} \exp \left(-\frac{\left(x-\mu_{l}\right)^{2}}{2 \eta_{l}^{2}}\right) d x
$$

Next, applying the result given in [34, Eq. (4)], i.e., $\mathcal{F}(x)=$ $\frac{1}{\left(1+\rho x^{2}\right)}$ is a real function of $x$, herein $y$ follows the normal distribution with mean $\mu_{l}$ and variance $\eta_{l}$, the expectation of $\mathcal{F}(x)$ can be highly approximated at three points, namely, $\mu_{l}$, $\mu_{l}+\sqrt{3} \eta_{l}$, and $\mu_{l}-\sqrt{3} \eta_{l}$ [35],

$$
\mathcal{E}[\mathcal{F}(x)] \approx \mathcal{F}\left(\mu_{l}\right)+\mathcal{F}\left(\mu_{l}+\sqrt{3} \eta_{l}\right)+\mathcal{F}\left(\mu_{l}-\sqrt{3} \eta_{l}\right) .
$$

Finally, substituting (10) into (5), the proof is accomplished.

\section{Outage Probability Characterization}

The outage probability is defined as the received SNR at the client vehicle $\gamma$ is below for a predetermined threshold $\gamma_{t h}$, mathematically speaking, $\mathcal{P}_{\text {out }}=\operatorname{Pr}\left(\gamma<\gamma_{t h}\right)=F_{\gamma}\left(\gamma_{t h}\right)$. Plugging (6a) into the definition of $\mathcal{P}_{\text {out }}$, yields

$$
\mathcal{P}_{\text {out }}\left(\gamma_{t h}\right)=\sum_{l=1}^{C} w_{l} \Phi\left(\frac{\sqrt{\gamma_{t h} / \rho}-\mu_{l}}{\eta_{l}}\right) .
$$

Remark 1. As stated earlier, the Fox's $H$-function distribution can be reduced to the Rayleigh distribution, i.e., $H_{0,1}^{1,0}(\mathbb{P})$, and $\mathbb{P}=(1,1,-, 0.5,-, 0.5)$. Using the CLT, we have that $Y$ follows the Gaussian distribution with mean $\frac{N \sqrt{\pi}}{2}$ and variance $\frac{N(4-\pi)}{4}$, i.e., $Y \sim \mathcal{N}\left(\frac{N \sqrt{\pi}}{2}, \frac{N(4-\pi)}{4}\right)$ [7]. Consequently, $\gamma=\rho Y^{2}$ is a non-central chi-square random variable with one degree of freedom. To this end, the outage probability at the client vehicle is

$$
\mathcal{P}_{\text {out }}\left(\gamma_{\text {th }}\right)=1-\mathcal{Q}_{\frac{1}{2}}\left(\sqrt{\frac{N \pi}{4-\pi}}, \sqrt{\frac{4 \gamma_{t h}}{\rho N(4-\pi)}}\right),
$$

where $\mathcal{Q}_{M}(a, b)$ is the Marcum $Q$-function.

Proof. Since $Y \sim \mathcal{N}\left(\frac{N \sqrt{\pi}}{2}, \frac{N(4-\pi)}{4}\right)$, then $Z=\frac{Y^{2}}{N(4-\pi) / 4}$ follows the non-central chi-square distribution with one degree of freedom and the noncentrality parameter $\frac{N \pi}{4-\pi}$. Applying the obtained results, one can obtain

$$
\begin{aligned}
\mathcal{P}_{\text {out }}\left(\gamma_{t h}\right) & =\operatorname{Pr}\left(\gamma<\gamma_{t h}\right)=\operatorname{Pr}\left(\rho Y^{2}<\gamma_{t h}\right) \\
& =\operatorname{Pr}\left(\frac{Y^{2}}{N(4-\pi) / 4}<\frac{4 \gamma_{t h}}{\rho N(4-\pi)}\right) \\
& =\operatorname{Pr}\left(Z<\frac{4 \gamma_{t h}}{\rho N(4-\pi)}\right),
\end{aligned}
$$

subsequently, applying the $\mathrm{CDF}$ of non-central chi-square distribution, the proof for $\mathcal{P}_{\text {out }}$ is obtained.

\section{Numerical Results AND Discussion}

In this section, we present the outage probability and the effective rate of the client vehicle for the two inter-vehicle communication scenarios, and the multipath fading model is drawn from Fox's $H$-function fading ${ }^{4}$, which is shown feasible and suitable for the $5 \mathrm{GHz}$ band inter-vehicle channels [19, Table IV],

- Scenario I: $h_{n} \sim H_{0,3}^{3,0}\left(\mathcal{P}_{1}\right)^{5}$,

$$
\mathcal{P}_{1}=\left(0.225,5.774,-,(1.5,0.4,4.5),-, \frac{1}{2} \mathbf{1}_{3}\right) .
$$

- Scenario II: $h_{n} \sim H_{0,3}^{3,0}\left(\mathcal{P}_{1}\right)$,

$$
\mathcal{P}_{2}=\left(2.874,3.940,-,(0.45,2,1.8),-,\left(\frac{1}{2} \mathbf{1}_{2}, 0.2\right)\right) .
$$

${ }^{4}$ The Fox's $H$-function distributed fading model is though widely studied in open literature as a general and unified model due to its flexibility of encompassing plenty of fading models, the implementation of generating of a Fox's $H$-function distributed random variable is found in [36].

${ }^{5} \mathbf{1}_{N}$ denotes the $N$-dimensional all-one vector. 


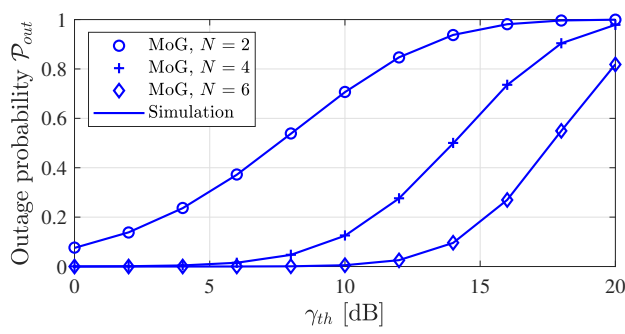

(a) Scenario I

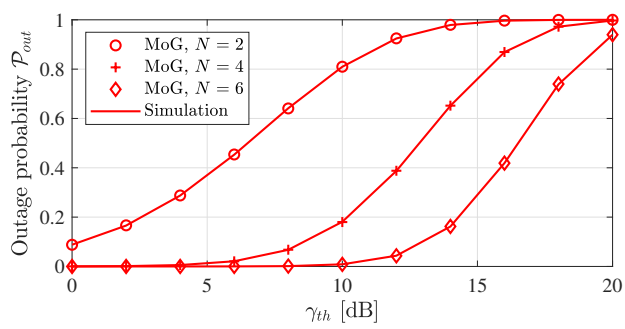

(b) Scenario II

Fig. 4: The outage probability of the RIS-assisted inter-vehicle communications against $\gamma_{t h}$ for selected values of $N$ when $\rho=10 \mathrm{~dB}$

In Fig. 4, we plot the outage probability against $\rho$ for the aforementioned two scenarios. The derived outage probability expression given in (11) provides a tight approximation to the simulated results.

Under the given two fading scenarios, Fig. 5 shows the effective rate against $\rho$ for the selected number of $N$. Outstandingly, one can grasp the following outcome: (i) our derivations provides a highly tight and accurate approximation to the Monte-Carlo simulated results; (ii) the effective rate is surely enhanced with the increase of $N$ as expected; (iii) observing from both Figs, the effect of an exponential rise of $N$ does not simply mean an unquestionably positive exponential improvement on $\mathcal{R}$. Apparently, only a linear increase trend of $\mathcal{R}$ is witnessed as $N$ is increasing from 32 to 64 , and finally arriving at 128; and (iv) this is a feasible application and proof showing that the MoG method is practical when performing performance analysis in the RIS-aided wireless systems [37].

In Fig. 6, the outage probability of our system configuration over Rayleigh fading channels is depicted for two cases, i.e., $N=2,3,4,5$ and $N=35,45,55$. The analytical result given from Remark 1 is compared with the MoG-based approximated result given in (8). The CLT-based result shows poor performance compared with the MoG-based analytical result, especially when $N$ is small, as shown in Fig. 6. (a). Besides, the MoG-based method always outperforms the CLTbased approach for both cases.

\section{CONCLUSION}

In this work, the IRS-assisted VANET was taken into consideration, where the inter-vehicle links are modeled with Fox's $H$-function distribution. Applying the MoG distribution, the instantaneous received SNR at the client vehicle

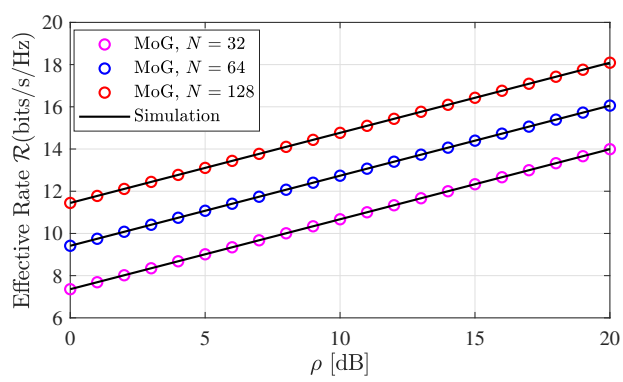

(a) Scenario I

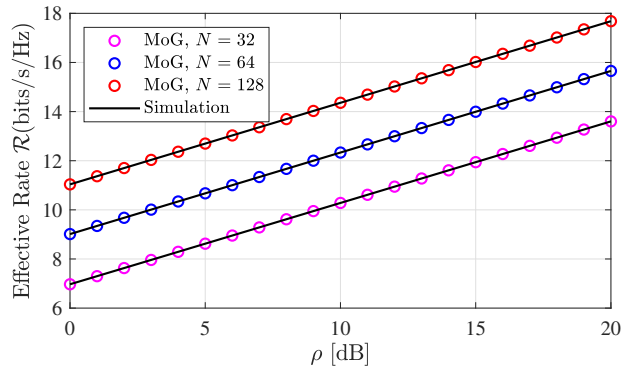

(b) Scenario II

Fig. 5: Effective rate against $\rho$ when $A=2$.

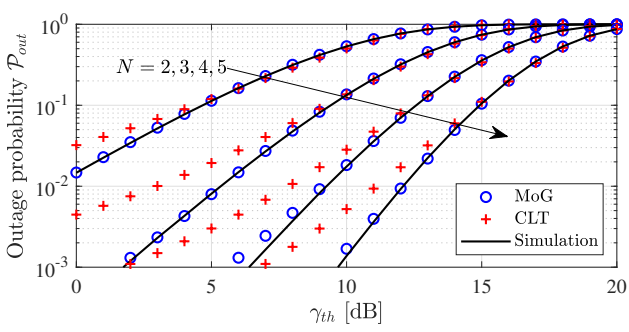

(a) $N=2,3,4,5, \rho=5 \mathrm{~dB}$

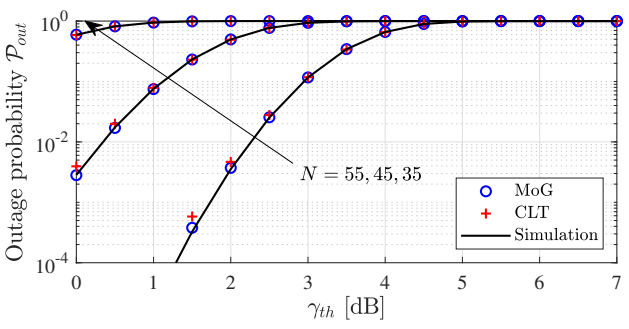

(b) $N=35,45,55$ and $\rho=30 \mathrm{~dB}$

Fig. 6: The outage probability against $\gamma_{t h}$ over Rayleigh fading channels for selected number of $N$.

is subsequently modeled as a MoG distributed RV, and the KS goodness of fit test further validates the accuracy of our approximated characteristics. Considering two fading channel scenarios, the outage probability and effective rate are explored as the performance metrics, which are derived with highly accurate closed-form expressions. Our numerical results showcased that (i) the obtained analytical performance metrics are firmly confirmed by the Monte-Carlo simulations; (ii) 
an increase of $N$ will not merely lead to a corresponding enhancement of effective rate; and (iii) the MoG-based method provides a highly accurate solution for the RIS-aided VANET link modeling for both small and large number of the reflector, and it outperforms the CLT-based method.

\section{ACKNOWLEDGMENT}

This work has been supported by the Luxembourg National Research Fund (FNR) projects, titled Exploiting Interference for Physical Layer Security in 5G Networks (CIPHY), and Reconfigurable Intelligent Surfaces for Smart Cities (RISOTTI).

\section{REFERENCES}

[1] Y. Chen, B. Ai, H. Zhang, Y. Niu, L. Song, Z. Han, and H. V. Poor, "Reconfigurable intelligent surface assisted device-to-device communications," early access, IEEE Trans. Wireless Commun., pp. 1-1, 2020.

[2] A. M. Elbir, A. Papazafeiropoulos, P. Kourtessis, and S. Chatzinotas, "Deep channel learning for large intelligent surfaces aided mm-Wave massive MIMO systems," IEEE Wireless Commun. Lett., vol. 9, no. 9, pp. 1447-1451, Sep. 2020

[3] J. He, H. Wymeersch, L. Kong, O. Silvén, and M. Juntti, "Large intelligent surface for positioning in millimeter wave MIMO systems," in IEEE VTC2020-Spring, Antwerp, Belgium, Belgium, 2020, pp. 1-5.

[4] X. Lu, E. Hossain, T. Shafique, S. Feng, H. Jiang, and D. Niyato, "Intelligent reflecting surface (IRS)-enabled covert communications in wireless networks," IEEE Network, vol. 34, no. 5, pp. 148-155, Sep./Oct. 2020.

[5] A. U. Makarfi, K. M. Rabie, O. Kaiwartya, X. Li, and R. Kharel, "Physical layer security in vehicular networks with reconfigurable intelligent surfaces," in IEEE VTC2020-Spring, Antwerp, Belgium, Belgium, 2020, pp. 1-6.

[6] Y. Ai, F. A. P. d. Figueiredo, L. Kong, M. Cheffena, S. Chatzinotas, and B. Ottersten, "Secure vehicular communications through reconfigurable intelligent surfaces," arXiv preprint arXiv:2011.14899, 2020.

[7] E. Basar, "Reconfigurable intelligent surface-based index modulation: A new beyond MIMO paradigm for 6G," IEEE Trans. Commun., vol. 68, no. 5, pp. 3187-3196, May 2020.

[8] S. Kisseleff, W. A. Martins, H. Al-Hraishawi, S. Chatzinotas, and B. Ottersten, "Reconfigurable intelligent surfaces for smart cities: Research challenges and opportunities," IEEE Open J. Commun. Soc., vol. 1, pp. 1781-1797, 2020.

[9] O. Özdogan, E. Björnson, and E. G. Larsson, "Intelligent reflecting surfaces: Physics, propagation, and pathloss modeling," IEEE Wireless Commun. Lett., vol. 9, no. 5, pp. 581-585, May 2020.

[10] M. Di Renzo, K. Ntontin, J. Song, et al., "Reconfigurable intelligent surfaces vs. relaying: Differences, similarities, and performance comparison," IEEE Open J. Commun. Soc., vol. 1, pp. 798-807, 2020.

[11] M. Di Renzo, A. Zappone, M. Debbah, et al., "Smart radio environments empowered by reconfigurable intelligent surfaces: How it works, state of research, and road ahead," IEEE J. Sel. Areas Commun., vol. 38, no. 11, pp. 2450-2525, Nov. 2020.

[12] E. Basar, M. Di Renzo, J. De Rosny, M. Debbah, M. Alouini, and R. Zhang, "Wireless communications through reconfigurable intelligent surfaces," IEEE Access, vol. 7, pp. 116753-116773, 2019.

[13] C. Huang, A. Zappone, G. C. Alexandropoulos, M. Debbah, and C. Yuen, "Reconfigurable intelligent surfaces for energy efficiency in wireless communication," IEEE Trans. Wireless Commun., vol. 18, no. 8 , pp. 4157-4170, Aug. 2019.

[14] Q. Wu and R. Zhang, "Beamforming optimization for wireless network aided by intelligent reflecting surface with discrete phase shifts," IEEE Trans. Commun., vol. 68, no. 3, pp. 1838-1851, Mar. 2020.

[15] L. Yang, Y. Yang, M. O. Hasna, and M. Alouini, "Coverage, probability of SNR gain, and DOR analysis of RIS-aided communication systems," IEEE Wireless Commun. Lett., vol. 9, no. 8, pp. 1268-1272, Aug. 2020.

[16] Q. Tao, J. Wang, and C. Zhong, "Performance analysis of intelligent reflecting surface aided communication systems," IEEE Commun. Lett., vol. 24, no. 11, pp. 2464-2468, Nov. 2020.
[17] L. Kong, Y. Ai, S. Chatzinotas, and B. Ottersten, "Effective rate evaluation of RIS-assisted communications using the sums of cascaded $\alpha-\mu$ random variates," IEEE Access, vol. 9, pp. 5832-5844, 2021.

[18] Y. Ai, M. Mohamed, L. Kong, A. Al-Saman, and M. Cheffena, "Hybrid automatic repeat request-based intelligent reflecting surface-assisted communication system," Electron. Lett., vol. 57, no. 7, pp. 303-305, 2021.

[19] Y. Jeong, J. W. Chong, H. Shin, and M. Z. Win, "Intervehicle communication: Cox-Fox modeling," IEEE J. Sel. Areas Commun., vol. 31, no. 9, pp. 418-433, Sep, 2013.

[20] D. P. Trinh, Y. Jeong, H. Shin, and M. Z. Win, "Molecular communication in H-diffusion," IEEE Trans. Commun., vol. 68, no. 7, pp. 42934310, Jul. 2020

[21] H. R. Alhennawi, M. M. H. E. Ayadi, M. H. Ismail, and H. A. M. Mourad, "Closed-form exact and asymptotic expressions for the symbol error rate and capacity of the $H$-function fading channel," IEEE Trans. Veh. Technol., vol. 65, no. 4, pp. 1957-1974, Apr. 2016.

[22] Y. Abo Rahama, M. H. Ismail, and M. S. Hassan "On the sum of independent Fox's $H$-function variates with applications," IEEE Trans. Veh. Technol., vol. 67, no. 8, pp. 6752-6760, Aug. 2018

[23] L. Kong and G. Kaddoum, "On physical layer security over the FisherSnedecor $\mathcal{F}$ wiretap fading channels," IEEE Access, vol. 6, pp. $39466-$ $39472,2018$.

[24] L. Kong, G. Kaddoum, and H. Chergui, "On physical layer security over Fox's $H$-function wiretap fading channels," IEEE Trans. Veh. Technol., vol. 68 , no. 7, pp. 6608-6621, Jul. 2019

[25] W. Aman, M. M. U. Rahman, S. Ansari, A. A. Nasir, K. Qaraqe, M. A. Imran, and Q. H. Abbasi, "On the effective capacity of IRS assisted wireless communication," arXiv preprint arXiv:2007.06825, 2020.

[26] C. Guo, L. Liang, and G. Y. Li, "Resource allocation for low-latency vehicular communications: An effective capacity perspective," IEEE J. Sel. Areas Commun., vol. 37, no. 4, pp. 905-917, Apr. 2019.

[27] F. Pernkopf and D. Bouchaffra, "Genetic-based EM algorithm for learning gaussian mixture models," IEEE Trans. Pattern Anal. Mach. Intell., vol. 27, no. 8, pp. 1344-1348, Aug. 2005.

[28] D. Wu and R. Negi, "Effective capacity: a wireless link model for support of quality of service," IEEE Trans. Wireless Commun., vol. 2, no. 4, pp. 630-643, Jul. 2003

[29] M. Amjad, L. Musavian, and M. H. Rehmani, "Effective capacity in wireless networks: A comprehensive survey," IEEE Commun. Surveys Tuts., vol. 21, no. 4, pp. 3007-3038, 4thquarter 2019.

[30] S. W. H. Shah, M. M. U. Rahman, A. N. Mian, A. Imran, S. Mumtaz, and O. A. Dobre, "On the impact of mode selection on effective capacity of device-to-device communication," IEEE Wireless Commun. Lett., vol. 8, no. 3, pp. 945-948, Jun. 2019.

[31] B. Selim, O. Alhussein, S. Muhaidat, G. K. Karagiannidis, and J. Liang, "Modeling and analysis of wireless channels via the mixture of Gaussian distribution," IEEE Trans. Veh. Technol., vol. 65, no. 10, pp. 8309-8321, Oct. 2016.

[32] L. Kong, S. Chatzinotas, and B. Ottersten, "Unified framework for secrecy characteristics with mixture of Gaussian (MoG) distribution," IEEE Wireless Commun. Lett., vol. 9, no. 10, pp. 1625-1628, Oct. 2020.

[33] J. Zhang, M. Matthaiou, G. K. Karagiannidis, and L. Dai, "On the multivariate Gamma-Gamma distribution with arbitrary correlation and applications in wireless communications," IEEE Trans. Veh. Technol., vol. 65 , no. 5, pp. 3834-3840, May 2016.

[34] G. Pan, C. Tang, X. Zhang, T. Li, Y. Weng, and Y. Chen, "Physicallayer security over non-small-scale fading channels," IEEE Trans. Veh. Technol., vol. 65, no. 3, pp. 1326-1339, Mar. 2016.

[35] J. M. Holtzman, "A simple, accurate method to calculate spreadspectrum multiple-access error probabilities," IEEE Trans. Commun. vol. 40, no. 3, pp. 461-464, Mar. 1992.

[36] Y. Aborahama, M. H. Ismail, and M. S. Hassan, "Novel methods for generating Fox's $H$-function distributed random variables with applications," IEEE Trans. Veh. Technol., vol. 68, no. 7, pp. 7150-7154, Jul. 2019.

[37] L. Kong, J. He, Y. Ai, S. Chatzinotas, and B. Ottersten, "Effective rate evaluation with assistance of mixture gamma (MG), mixture of gaussian (MoG), and Fox's $H$-function distributions," in IEEE VTC2021-Spring, Helsinki, Finland, 2021, pp. 1-6. 